\title{
COMPARANDO SISTEMAS DE INNOVACIÓN: UNA REVISIÓN DE LA LITERATURA SOBRE ALEMANIA Y CHINA
}

COMPARANDO SISTEMAS DE INNOVACIÓN: UNA

REVISIÓN DE LA LITERATURA SOBRE ALEMANIA Y CHINA

Fecha de recepción: 15 de noviembre de 2018.

Daniel F. Crespo Pazmiño ${ }^{1}$

Fecha de aceptación: 11 de enero 2019.

(dfcrespofl@flacso.edu.ec)

Código JEL: B52; 000.

Código D0I: 10.25097/rep.n29.2019.05

\section{Resumen}

La globalización, el creciente desarrollo tecnológico y la capitalización del conocimiento, han reposicionado a la innovación como el componente de competitividad clave para el desarrollo y bienestar económico de Estados y empresas. Por dicha razón, existe un creciente interés por el estudio de las dinámicas y políticas que influyen en el desarrollo y aplicación del proceso innovador.

El presente trabajo, mediante una revisión literaria, expone las aproximaciones metodológicas utilizadas dentro de estudios empíricos de comparación de sistemas nacionales y regionales de innovación de Alemania y China. En ambos casos, se evidencia que el estudio académico contemporáneo de la innovación toma lugar desde una perspectiva evolutiva, se enfoca en las vinculaciones entre los diferentes agentes e instituciones y su análisis contribuye a la estrategia para el establecimiento de políticas a favor del desarrollo económico y bienestar social.

Palabras clave: innovación, metodología, política tecnológica, revisión de literatura, sistemas de innovación.

\section{Abstract}

Globalization, the growing technological development, and the capitalization of knowledge, have repositioned to innovation as the key competitive component for economic development and welfare of states and companies. Hence, there is a growing interest in the study of the dynamics and policies that influence the development and implementation of the innovative process.

This paper, through a literary review, presents the methodological approaches used within empirical studies comparing the national and regional innovation systems of Germany and China. In both cases, there is evidence that the contemporary academic study of innovation takes place from an evolutionary perspective, it focuses on the linkages between the different actors and institutions in a system, and its analysis contributes to the strategy used in the establishment of public policies to favor economic development and social welfare.

Keywords: innovation, methodology, technology policy, literature review, innovation systems.

1 Maestria de Relaciones Internacionales con mención en Seguridad y Derechos Humanos, Facultad Latinoamericana de Ciencias Sociales (Flacso) - Ecuador, ORCID: 0000-0002-9532-2672 


\section{Introducción}

La globalización, el creciente desarrollo tecnológico y la capitalización del conocimiento, han reposicionado a la innovación como el componente de competitividad clave para el desarrollo y bienestar económico de Estados y empresas (Fagerberg, Martin y Andersen, 2013; Ordóñez, 2017). Esto se debe a que la llegada del proceso globalizador, generó un cambio del paradigma de las relaciones políticas, socioeconómicas y tecnológicas a nivel mundial que posicionó al conocimiento como el recurso fundamental de la economía moderna y a la innovación como su proceso de aplicación práctica (Kirshner, 2009; Lundvall, 2017). De esta forma, el estudio, desarrollo y aplicación de la innovación en el ámbito de la globalización, adquiere un mayor rol en las esferas académica, profesional y política (Soete, Verspagen y ter Weel, 2010; Lundvall, 2017).

De esta forma, el estudio de la innovación permite a los tomadores de decisión mejorar sus consideraciones en la planificación estratégica de políticas fiscales o empresariales, enmarcadas en escenarios locales, nacionales, regionales y mundiales (Edquist, 2005). No obstante, si de comparar analíticamente los sistemas de innovación (SI) y las estrategias de diferentes países se trata, existe una relativa baja teorización de dicho enfoque. Por ejemplo, al revisar literatura de estudios comparados de innovación sobre los diez países que encabezan el posicionamiento económico mundial para 2018, se evidencia una predisposición de estudios comparativos entre Estados Unidos y otro Estado de la lista, más no entre las demás economías top mundiales. Así, se observa la necesidad de ampliar el campo de estudio mediante la comparación analítica de países con circunstancias disímiles.

Es así que nace el presente trabajo, el cual tiene como objetivo evidenciar las aproximaciones teóricas y metodológicas usadas en estudios empíricos de SI sobre Alemania y China. Para 2017, dichas economías se posicionan fuertemente (política y económicamente) en el entorno global, a pesar de contar con trayectorias y estrategias de producción dispares. Por un lado, Alemania posee una relativamente amplia historia vinculada con la integración económica, así como, una estrategia de producción económica de cuarta generación, conocida como industria 4.0, la cual se centra en desarrollo tecnológico de punta (Miguelez, 2017). Por otro lado, China cuenta con una relativamente corta trayectoria de integración económica, así como con una estrategia de producción industrial de segunda y tercera generación, la cual recurre principalmente a la producción en masa de productos previamente desarrollados en otros países (Wübbeke et al., 2016).

De esta forma, el presente artículo inicia con una revisión literaria que explica y articula nociones básicas como la globalización y conceptos en torno a los que se desarrollan los principales debates teóricos sobre la innovación. Igualmente, se establecen las posturas en cuanto al estudio y los límites, dificultades y oportunidades al comparar sistemas nacionales de innovación, se vinculan con los casos disimiles seleccionados de Alemania y China, y se presentan conclusiones al respecto.

\section{Revisión de la literatura}

La globalización es un proceso que ha intensificado la interdependencia de los Estados al acelerar el fenómeno de internacionalización comercial propio del capitalismo (Kirshner, 2009; Keohane y Nye, 2012). La globalización responde a una lógica de interconexión, reducción de distancias, fronteras y del tiempo entre ellas, a causa del rápido desarrollo tecnológico y de 
las telecomunicaciones. En consecuencia, el proceso impacta en aspectos sociales, políticos, culturales y de desarrollo tecnológico (Keohane y Nye, 2012). Esto, además, implica un proceso espacial de re jerarquización de escalas geográficas y la consolidación del conocimiento como recurso, o la denominada capitalización del conocimiento (Kirshner, 2010; Ordoñéz, 2017).

Respecto al primero, el nivel de enfoque nacional comienza a perder relevancia relativa frente al incremento de importancia de las escalas locales que se vinculan directamente con escalas transnacionales y supranacionales (Ordoñéz, 2017). Esto se debe, en parte, al cambio en la dinámica de regionalización, lo cual pasa de ser un ordenamiento territorial nacional y regional (arriba-abajo), a un orden de relación mixta (abajo-arriba y arriba-abajo) en el que lo local y lo global coexisten directa e independientemente y se establece un nuevo regionalismo (Vivares, Torres P y Cvetich, 2014).

Respecto al conocimiento, este pasa a constituirse como el recurso fundamental de la economía moderna o capital intelectual y se enmarca en una lógica de productiva (circulación, acumulación y aplicación del conocimiento) aprovechada para la generación de valor en la producción económica (Fagerberg, 2005; Lundvall 2017; Ordoñéz, 2017). Este recurso se nutre del proceso de aprendizaje, el cual es de naturaleza interactiva y social, y no es posible entender sin un contexto cultural e institucional (Mokyr, 2010; Lundvall 2017). Lundvall (2017) denomina a dicho proceso innovación y lo enmarca en una lógica de interacción sistémica para analizar su contribución de la generación de conocimiento al entorno económico productivo.

El contexto dentro del cual se entiende la innovación difiere histórica, cultural e institucionalmente. Así, existen tres grandes enfoques desde los cuales se explica el fenómeno: el neoclásico, el neoschumpeteriano y el de modelos de partida (Fagerberg, Martin y Andersen, 2013; Sastre 2015; Ordóñez, 2017). Primero, la visión neoclásica se basa en la visión del capitalismo, de la mano invisible y corrección de fallos del mercado (Ordóñez, 2017). En ella, la relación del proceso de aprendizaje se consolida en una relación de arriba-abajo, donde la innovación es vista como un proceso lineal y secuencial que resulta en un elemento exógeno de fácil difusión y copia (Freeman, 1987). Además, tiene una visión homogeneizadora de los actores del sistema y bajo una lógica de competencia perfecta. Como resultado, las empresas no se encuentran directamente motivadas para invertir en procesos de I+D y el Estado debe establecer políticas para incentivar dicho proceso (Freeman, 1987; Fagerberg, 2005).

Segundo, desde la visión neoschumpeteriana se presenta un enfoque estructuralistaevolucionista donde la innovación resulta de articulación entre ciencia, educación y el proceso producción económico-social (Mokyr, 2010). La corriente evolutiva, estudia la innovación en el marco de las instituciones y en un entorno de continuo cambio e interacción a nivel sistémico, mismo que está caracterizado por la incertidumbre (Freman, 1987; Sastre 2015). Esto se debe a que los actores involucrados en el proceso innovador - principalmente empresas- manejan una "racionalidad limitada" por lo cual es poco probable tomar en consideración todos los factores del entorno económico para la toma de decisiones (Edquist, 2004; Lundvall, 1992; Nelson y Rosenberg, 1993).

Esta visión además, considera a las empresas como unidades diferenciadas por sus estrategias, estructura y rutinas (Verspagen, 2005; Sastre, 2015). Por tanto, las estrategias que las empresas utilizan para innovar -las cuales pueden ser de tipo ofensivas, defensivas, imitativas, dependientes, oportunistas o tradicionales-condicionan la aproximación con la que un actor ve el desarrollo de conocimiento, la innovación y tecnología (Fagerberg, Martin y Andersen, 2013). Respecto a la visión del SI, en el evolucionismo se compara al sistema con un ser vivo el cual 
se encuentra bajo constante retroalimentación y evolución. Por ello, el SI constituye la red de organizaciones e instituciones, tanto públicas como privadas, que a través de su interacción dan lugar a la creación, importación, modificación, difusión y uso de conocimiento, y este último es entendido como tecnología (Freeman, 1987; Edquist, 2004; Soete et al, 2010; Sastre, 2015).

Tercero, la visión de modelos de partida, también vistos como modelos enfocados en resultados de articulación técnico-operativa, divisa a los SI bajo un enfoque que analiza elementos del entorno que generan y acumulan conocimiento de manera más individual a manera de determinar en sus relaciones y límites (Ordoñez, 2017). Aquí, el papel protagónico del Estado, característico de las visiones precedentes, se va diluyendo al sufrir la equiparación del papel de otros actores estatales o no estatales en cuanto a relaciones de interdependencia e incentivos (Ordoñez, 2017). Además, este enfoque se relaciona más con el del nuevo regionalismo. Por tanto, su énfasis se establece con la noción neoliberal del capitalismo y el enlace local y global en un solo espacio geográfico. Quitero-Campos (2010) y Ordoñez (2017) identifican tres modelos de sistemas nacionales de innovación de este último:

- El modelo de entornos de Fernández de Lucio y Conesa (1996) caracterizado por la interacción de cuatro entornos: científico (universidades), tecnológico (centros servicios tecnológicos), productivo (parques tecnológicos) y financiero (entidades de capital de riesgo)

- El modelo de Triple hélice (1996): el cual considera al Estado, Empresa y Universidad como unidades independientes que se organizan por objetivos puntuales. Este cuenta con dos modos o enfoques: el primero el Estatal coordinador, similar a la estructuración evolucionista y laissez faire del neoclásico.

- El modelo de Cotec (2005): en este la empresa ocupa lugar central y se apoya en los demás elementos, estos son: a) entorno, b) sistema público de $\mathrm{I}+\mathrm{D}+\mathrm{i}, \mathrm{c}$ ) infraestructuras, d) administración. En este modelo se hace una considerable reducción de la acción del Estado y se eleva al mercado como institución coordinadora de interacción entre agentes o entornos.

Con relación a las perspectivas de análisis sobre SI, estos pueden entenderse desde una visión territorial-espacial, que se divide en nacional, sectorial, regional y global (Edquist, 2004; Quitero-Campos, 2010 Sastre, 2015). El enfoque nacional -al estar dotado de una delimitación espacial, un sistema de gobierno e indicadores macro medibles y comparables en el tiempoes útil al examinar el papel del Estado en aspectos macro (Arocena y Sutz, 2002; Sastre, 2015). Sin embargo, tal y como destacan Liu y White (2001), el enfoque de análisis nacional ha sobrellevado críticas sobre la generalización de la perspectiva estatal y sobre el valor de un análisis gubernamental en un mundo cada vez más globalizado, donde los enlaces y los flujos de información superan las fronteras políticas estatales.

Ante esto, Lundavall (2016) explica que la fortaleza de esta aproximación espacial se debe a que los procesos de aprendizaje, incluso en la dinámica del regionalismo y la globalización, se encuentran asentados dentro de una noción territorial Estatal. Por ello, estudiar la innovación con la limitante de una consideración política, su jurisdicción e instituciones directamente vinculadas, ayuda a una comprensión sólida del entorno de innovación.

Quitero-Campos (2010), por su parte, evidencian la relevancia del enfoque regional para el estudio de SI con clústers de desarrollo de conocimiento específicos y que tienen a una lógica de nuevo regionalismo, la cual añade una visión de heterogeneidad intranacional. Esta noción 
adicional es relevante en el estudio de la interacción interna del sistema nacional de innovación como ente compuesto (Ordoñez, 2017). Por tanto, una configuración mixta de sistema nacionalregional de innovación (SN-R_I) permite mantener un margen de enfoque maleable en cuanto a Estados de soberanía unitaria como el caso de China y de soberanía distribuida en federativos como en Alemania.

Finalmente, para este nivel mixto, se plantea identificar los $\mathrm{SI}$ en función del grado de interrelación entre actores, siendo este emergente o maduro. (Chaminade et al., 2010). Así, el SI emergente está caracterizado por su baja articulación entre actores del sistema y tiende a estrategias de innovación empresarial más imitativas, tradicionales y dependientes; mientras que el SI maduro evidencia complejas redes de interconexión entre agentes. Esto permite una transmisión de conocimiento eficiente y favorable para estrategias de innovación ofensivas y defensivas (Edquist, 2004; Chaminade et al., 2010).

\section{Materiales y Métodos}

El presente documento hace uso de una revisión de la literatura científica existente sobre los sistemas nacionales y regionales de innovación (SN-R_I) de Alemania y China, enfatizando en la metodología aplicada en dichos trabajos empíricos. Para ello, primero se utilizó una revisión sistémica exploratoria y analítica de revistas indexadas y libros más destacados sobre el tema con método deductivo. A continuación, a través del método inductivo, se procedió a comparar y sintetizar la información más relevante para establecer el estado del estudio de SI en Alemania y China, en relación a la teoría evolutiva neoschumpeteriana y de enfoque en resultados.

De esta manera, tras la revisión exhaustiva de fuentes, se identificó seis artículos que estudian empírica y analíticamente los sistemas nacionales y regionales de innovación de Alemania y China. A ellos se suman artículos y libros secundarios que estudian la innovación y el cambio tecnológico en los países de foco (China y Alemania) pero no a nivel sistémico. Así, la literatura adicional sobre innovación en China y Alemania dialoga en torno a los seis estudios empíricos base (ilustrados en la Tabla 1) que tratan la dinámica de SN-R_I. Para ello, se presenta una revisión de trabajos principales divididos en análisis individuales y comparados, y ordenados por país y cronológicamente.

Tabla 1. Estudios empíricos de SN-R_I de Alemania y China analizados

\begin{tabular}{|l|l|l|l|l|}
\hline & Año & Tipo de estudio / Enfoque & Tema & Autor \\
\hline ALEMANIA & 1993 & Individual / histórico-Institucional & $\begin{array}{l}\text { The National System for Technical } \\
\text { Innovation in Germany }\end{array}$ & Otto Keck \\
\cline { 2 - 6 } & 2011 & Comparado / Actores y políticas & $\begin{array}{l}\text { Research and Innovation Policy in } \\
\text { the U.S. and Germany: a Compa- } \\
\text { rison }\end{array}$ & $\begin{array}{l}\text { Carla Hom- } \\
\text { mes, Anselm } \\
\text { Mattes, Do- } \\
\text { reen Triebe }\end{array}$ \\
\cline { 2 - 6 } & 2015 & Individual / Histórico-Institucional & $\begin{array}{l}\text { The National Innovation System in } \\
\text { Germany }\end{array}$ & $\begin{array}{l}\text { Matthew } \\
\text { Allen }\end{array}$ \\
\hline
\end{tabular}




\begin{tabular}{|c|c|c|c|c|}
\hline \multirow[t]{5}{*}{ CHINA } & \multirow[t]{2}{*}{2001} & \multirow{2}{*}{$\begin{array}{l}\text { Individual - Comparado* / } \\
\text { Histórico - enlaces y Actividades } \\
\text { principales }\end{array}$} & $\begin{array}{l}\text { Comparing innovation systems: a } \\
\text { framework and application }\end{array}$ & \multirow[t]{2}{*}{$\begin{array}{l}\text { Xielin Liu y } \\
\text { Steven White }\end{array}$} \\
\hline & & & to China's transitional context & \\
\hline & 2009 & $\begin{array}{l}\text { Comparado / Políticas y Subsiste- } \\
\text { mas }\end{array}$ & $\begin{array}{l}\text { The Comparison of China and India: } \\
\text { National Innovation System }\end{array}$ & $\begin{array}{l}\text { Wu Xiaobo, } \\
\text { Aromar Revi } \\
\text { y Hiren Doshi }\end{array}$ \\
\hline & \multirow[t]{2}{*}{2016} & \multirow[t]{2}{*}{$\begin{array}{l}\text { Comparado / Actores, políticas y } \\
\text { enlaces }\end{array}$} & $\begin{array}{l}\text { National Innovation Systems in the } \\
\text { United States and China: }\end{array}$ & \multirow{2}{*}{$\begin{array}{l}\text { Aaron Me- } \\
\text { laas y Fang } \\
\text { Zhang }\end{array}$} \\
\hline & & & A Brief Review of the Literature & \\
\hline
\end{tabular}

Nota*: El artículo de Liu y White (2001) compara el sistema de innovación de China antes y después de las reformas de transición nacional económica.

Fuente: documentos revisados descritos.

Elaboración: el autor.

\section{Discusión y resultados}

\subsection{Sistema de innovación en Alemania}

Tanto el de Keck (1993) como el de Allen (2015), distinguen a las políticas estatales de vinculación entre empresas y centros de investigación como los ejes de la capacidad tecnológica y competitiva industrial del SI alemán. Ambos análisis detallan las condiciones históricas y cambios en las políticas estatales a nivel educativo e industrial que fomentaron avances en los procesos de desarrollo tecnológico a través de investigación especializada; lo que dio paso a su SN-R_I actual. Para ello, en una primera parte, se examinan las raíces del SN-R_I alemán en la creación y fomento de universidades y centros de investigación en el siglo XIX, así como el desarrollo industrial inicial de empresas eléctricas y químicas (Keck, 1993; Allen, 2015). Como segunda parte, se estudia el papel de la investigación y desarrollo en la industria luego de la unificación de Alemania y las políticas y metas tecnológicas que alimentan el SN-R_I actual.

Respecto a las raíces del SN-R_I de Alemania, Keck (1993) y Allen (2015) destacan el papel de las políticas públicas en direccionar el trabajo de las universidades hacia la investigación, así como a la promoción de centros de investigación especializados, mediante incentivos económicos y fondos de investigación desde el siglo XIX. De igual manera, bajo la lógica de innovación desde la visión neoschumpeteriana y de enfoque en resultados enfatizada por Thelen (2004) y Allen (2015), se destacan los debates académicos y esfuerzos públicos y privados por generar canales de vinculación entre las universidades y las empresas, a manera de una integración del entorno científico, tecnológico, productivo y financiero (Thelen, 2004; Allen, 2015). Esto dio como resultado la generación de industria tecnológica que encontraba su ventaja competitiva en el alto grado de especialización de sus procesos de investigación y la búsqueda continua de eficiencia en dicho proceso de manera sistemática, tanto sectorial y regional, como nacional (Keck 1993; Culpepper y Finegold, 1999; Allen 2015).

Para fines del siglo XIX y desarrollo del siglo XX, dentro de los principales sectores de énfasis de la investigación de universidades y empresas en Alemania se posicionan: el desarrollo de la investigación, las industrias química-farmacéutica, automotriz, electrónica, de construcción y minera (Keck 1993; Culpepper y Finegold, 1999). Dicho énfasis por centros de investigación fue posible gracias a la descentralización de competencia sobre las universidades a nivel federal y 
la asistencia financiera institucional (Whitley, 2007; Allen, 2015). De esta forma, cada uno de los Estados federales de Alemania enfocaba sus políticas y reformas de educación e innovación en función de las capacidades de producción principales de cada región, así como de los sectores de desarrollo tecnológico donde existía mayor probabilidad de lograr innovaciones (Keck 1993; Allen 2015).

Con relación al papel de la investigación, el desarrollo industrial y las políticas tecnológicas que alimentan el SNI contemporáneo, los estudios muestran que el sistema de investigación está perfilado por centros de investigación tecnológica especializados que trabajan en estrecha vinculación con el sector industrial (Whitley, 2007; Allen 2015). Además, luego de la reunificación, la competitividad alemana se centra en industrias de alta tecnología de tamaño medio y pequeño que atienden nichos de mercado en sectores específicos (Keck 1993; Thelen, 2004; Allen 2015). Por ende, las instituciones clave en el SN-R_I de Alemania son: primero, el sistema político, conformado por el gobierno federal, el gobierno central y la Unión Europea; segundo, las organizaciones intermediarias, mismas que dirigen proyectos de organización y asociaciones industrial y de soporte financiero; y, tercero, el sector de desarrollo de investigación, el cual está conformado por entidades públicas y privadas dedicadas a la investigación y desarrollo (I+D) (Keck 1993; Culpepper y Finegold, 1999; Allen 2015).

Dentro del sector de investigación se destaca el rol del sistema educativo alemán, el cual cuenta con tres entornos de aprendizaje: (1) universidades de estudios teóricos y científicos, (2) universidades y centros de investigación especializados en administración y negocios; y (3) entornos de aprendizaje práctico bajo el sistema dual (una modalidad de estudio y practica laboral específica) (Hommes, Mattes y Triebe 2011; Kroll y Frietsch, 2010; Allen 2015). Estos tres enfoques de educación en torno al desarrollo de investigación, junto con la praxis, dan lugar a la generación de clústers que ayudan a vincular la innovación con los procesos económicos cotidianos, lo que, en conjunto, mejora la inserción de profesionales. Además, diversos proyectos de I+D cuentan con incentivos para que investigadores y empresas continúen generando innovación en sectores competitivos de interés público (Keck 1993; Hommes, Mattes y Triebe, 2011; Allen 2015).

\subsubsection{Comparando el SN-R_I de Alemania con Estados Unidos}

En cuanto a estudios de comparación del SN-R_I sobre Alemania, Hommes, Mattes y Triebe (2011) realizan un análisis comparado pionero de políticas de innovación con Estados Unidos. El artículo hace una discusión teórica de la I+D con una visión evolutiva que se enfoca en las políticas estatales que ambos países han adoptado para mejorar los enlaces entre actores del SI y sus objetivos en cuanto a futuro desarrollo tecnológico. Igualmente, en dicho estudio se compara el entorno nacional, las instituciones rectoras de la innovación y el rol de los principales actores al momento de realizar procesos de I+D.

Aunque el trabajo de Hommes, Mattes y Triebe (2011) toma en consideración puntos clave del SNR_I vistos por Keck (1993) y Allen (2015) en sus análisis individuales, el estudio difiere en la forma en que la innovación es desarrollada por las políticas estatales, las cuales consideran similitudes, peculiaridades, fortalezas y debilidades de SN-R_I de Alemania y Estados Unidos. Con ello, los autores evidencian que pese a que las vinculaciones entre los actores y organizaciones del SNR_I alemán son mucho más complejas y maduras que en el SN-R_I estadounidense, Alemania aún tiene pasos que recorrer en cuanto al desarrollo de universidades de alto grado tecnológico (Hommes, Mattes y Triebe, 2011). Se destaca también que la sociedad americana cuenta con un sistema legal y financiero más abierto al espíritu emprendedor, lo cual la vuelve más dinámica que la sociedad alemana en cuanto al proceso de destrucción creativa y de generación de 
innovaciones en nuevas áreas de conocimiento (Fagerberg, 2005; AICGS, 2006; Hommes, Mattes y Triebe 2011).

Con relación a indicadores, el estudio de comparación hace uso de datos macro manejados por los ministerios a cargo de la coordinación de la actividad innovadora. Se resalta el número de universidades y centros de investigación públicos y privados, así como el desenvolvimiento y destino de la inversión doméstica en I+D. Asimismo, el análisis toma como elemento base los rankings pertenecientes a la Fundación de Tecnologías de Información e Innovación (ITIF) y el Índice Global de Innovación (GIL). De esta forma se presenta una evaluación general de inputs y outputs de la inversión en I+D -la cual evidencia la eficiencia de la inversión en I+D en relación al PIB- y se encuentra en niveles comparables de aproximadamente $2.5 \%$, pero se diferencia en las instituciones destino (Hommes, Mattes y Triebe 2011; Sastre 2015). Así pues, en Estados Unidos la inversión se concentra más en sectores de innovación de tipo más radical y básica como en nanotecnología y robótica. Por otra parte, en Alemania se prioriza la innovación de tipo incremental en los sectores especializados de su economía -como son la química y farmacéutica y la industria automotriz (Hommes, Mattes y Triebe 2011).

\subsection{Sistema de innovación en China}

En cuanto a los ensayos académicos que tratan analíticamente el SN-R_I de China - Liu y White (2001), Wu, Revi y Doshi (2009) y Melaas y Zhang (2016) -, estos son en esencia trabajos de comparación de SN-R_I de China con otro Estado, con la excepción del trabajo de Liu y White (2001) que compara la evolución de China tras reformas económicas. En dichos estudios se presenta la significativa evolución económica que sufrió la nación China, junto con los desafíos estatales que el país enfrenta al tratarse de una nación con creciente auge comercial que todavía se encuentra en una fase emergente de su SN-R_I. La revisión del SN-R_I en China, por tanto, se centra en la comparación de metodologías usadas para tratar y contrastar el SI chino, las contribuciones de cada uno y los desafíos pendientes para próximos análisis.

\subsubsection{Metodología de estudios comparados del SN-R_I de China}

En primer lugar, los tres trabajos comparten una visión de innovación neoschumpeteriana con un tinte de la visión de enfoque en resultados, motivo por el que cada uno de ellos resalta el papel del Estado en generar vinculaciones entre sectores para mejorar la transmisión de conocimiento y, en consecuencia, favorecer al desarrollo de la innovación tecnológica en la industria de producción china (Liu y White, 2001; Wu, Revi y Doshi, 2009; Fagerberg, Martin y Andersen, 2013; Sastre 2015; Melaas y Zhang, 2016). Al igual que los estudios del SN-R_I de Alemania, los documentos muestran la tendencia académica para tratar la innovación desde una visión tanto evolutiva, como de enfoque en resultados, mismas que superan varias limitaciones de la perspectiva neoclásica (Liu y White, 2001; Sastre, 2015; Ordóñez, 2017). Dentro de los documentos se distinguen tres visiones de comparación de SN-R_l: a) una visión descriptiva sobre el funcionamiento y enlaces institucionales de cinco actividades fundamentales para la innovación, en concordancia con el modelo estructural-evolucionista; b) una perspectiva sistémica del entorno económico y sus resultados, propia del modelo de agentes de Fernández de Lucio y Conesa; y, c) un análisis sobre el papel de los actores, las políticas estatales y los enlaces institucionales, característico del modelo general neoinstitucionalista.)

En la primera metodología, planteada por Liu y White en 2001, se observa un esfuerzo por superar las críticas y dificultades tradicionales al analizar SN-R_I establecidos por economistas neoschumpeterianos, como Nelson (1993) o Lundvall (1992; 2016), las cuales hacen referencia 
a la visión neoclásica de la innovación como resultado endógeno y a los límites de comparación sobre el espacio geográfico de referencia. Se destaca también que los estudios de SI se limitan a economías similares en términos de producción industrial (Patel y Pavitt, 1998; Liu y White, 2001). Esto dificulta la comparación entre SI en fases emergentes -como es el caso de los países en desarrollo como China- y SI maduros -como es el caso de economías avanzadas como la de Estados Unidos. A partir de la necesidad de contar con un modelo de comparación sistemático sobre los enlaces entre instituciones y actividades productivas, Liu y White (2001) proponen centrarse tanto en las vinculaciones de los actores principales y secundarios del SI basados en las sugerencias de Anderson y Lundvall (1997). Para ello, se sugiere un análisis desde cinco actividades de innovación fundamentales: I+D, implementación, uso final, educación y vinculación. Así, el marco de análisis y comparación de SN-R_I permite realizar un contraste sobre la evolución de la estructura, sus dinámicas y su desempeño en el sistema.

Dentro de la segunda metodología, utilizada por Wu, Revi y Doshi (2009), se evidencia una aproximación sistémica parecida a la de Liu y White. No obstante, los autores buscan simplificar la dinámica y la comparación misma al enfocarse en las políticas y sus resultados en tres subsistemas de China e India: el subsistema de ambiente innovador, el subsistema de operación innovadora y el subsistema de desempeño económico. Respecto al entorno de innovación, se analizan las instituciones que realizan y coordinan actividades de investigación, como el Ministerio de Ciencia y Tecnología y las universidades públicas. Sobre el subsistema de operación de las innovaciones, aquí figuran los inputs y outputs de la inversión en I+D a nivel nacional, como es el caso de la inversión en centros de investigación e investigadores (Fagerberg, Srholec y Verspagen, 2010). Este subsistema es medido con el número de patentes y artículos técnicos generados a partir de dicha inversión. En cuanto al subsistema de resultados económicos, en él se cataloga la evolución del incremento económico en términos generales a nivel de crecimiento del PIB y, además, se evalúa la complejidad de la estructura industrial puesta en marcha (Wu, Revi y Doshi, 2009; Abdi y Aulakh, 2012).

Referente a la tercera metodología utilizada por Melaas y Zhang (2016), se consideran los marcos de análisis tradicionales como en Nelson (1993), el propuesto por Liu y White (2001) y dificultades metodológicas al comparar economías de diferentes niveles reveladas por Carlsson, et al. (2002). En ese sentido, los autores resaltan las diferencias a tomar en consideración al analizar un SI como el de China, dado que la nación sigue siendo una economía en desarrollo que enfrenta desafíos de desarrollo socio-económicos, como la inequidad evidenciada por la gran cantidad de habitantes en zonas rurales y el elevado grado de industrialización polarizada en pocas zonas (Carlsson, et al. 2002; Liu, Han y Chen, 2014; Melaas y Zhang, 2016).

De esta manera, los autores proponen un estudio comparado de SN-R_I que integra consideraciones neo-institucionalistas y de enfoque a resultados con base en tres ejes. El primero se refiere a las políticas de apoyo a la innovación del sector público y privado. Aquí se comparan los sistemas de políticas de China y Estados Unidos en cuanto al apoyo directo o indirecto a la innovación. El segundo trata las políticas y desafíos de la investigación básica y aplicada en centros de I+D, en donde se evalúan prioridades, vinculaciones entre actores y la respuesta a los incentivos económicos para la I+D. Finalmente, en el tercer apartado se evalúan los objetivos de las políticas de innovación y sus resultados. Para tal fin, se exploran las estrategias empresariales de innovación de Estados Unidos y China -siendo la una de tipo radical y la otra imitativa (Fagerber, Mowery y Nelson, 2005; Carey, Hill y Kahin, 2012). Igualmente, se estudia el estado de la vinculación nacional con multinacionales y clústers internacionales, a partir de procesos de formación educativa y laboral (Melaas y Zhang 2016). 


\subsubsection{Contribuciones y desafíos de los estudios de SN-R_I de China}

Dentro de la revisión de los tres trabajos principales sobre el SN-R_I en China, se evidenció como aspecto principal el cambio de políticas económicas gubernamentales de una economía planificada a una economía de mercado, lo que mejoró significativamente la vinculación entre instituciones dedicadas al desarrollo de la I+D. Así también, se resalta el papel de la industria y las universidades en generar innovación tecnológica e industrial dentro de un sistema aún en fase inicial pero de rápido crecimiento y apertura a la globalización (Chen, 2009; Guo y Guo, 2011). De igual forma, otro elemento crucial en el cambio de la innovación en China se debe a creciente integración económica regional y mundial a través de la presencia de multinacionales y del proceso de ingreso de al GATT/OMC(Liang, 2002; Chen, 2009; Cai, Cui y Pugh, 2015; Melaas y Zhang 2016). Estas, atraídas por la nueva apertura económica y el mercado de trabajo significativamente barato, permiten el desarrollo de empresas e industria interna, tanto por la transferencia de conocimiento formal y tácito sobre los bienes manufacturados -en el caso de alianzas-, como por la aparición de firmas que facilitan el trabajo de las multinacionales (Todo, Zhang y Zhou, 2006; Chen, 2009; De la Tour, Glachant y Ménière, 2011; Castellani, Jimenez y Zanfei, 2013; Gallagher y Zhang, 2016).

Sin embargo, aunque existe un crecimiento en la innovación tecnológica desarrollada en China, esta dinámica está en etapas iniciales en comparación con Estados Unidos; motivo por el cual existe una fuerte tendencia a adquirir tecnología y darle un ligero desarrollo en términos de adaptación (Melaas y Zhang 2016). Asimismo, pese al establecimiento de incentivos económicos en el proceso de I+D, la nación china continúa con un baja capacidad sistémica de incentivar actividades de riesgo en la innovación (Castellani, Jimenez y Zanfei, 2013; Melaas y Zhang 2016). Otro desafío del SN-R_I chino es la mejora del entorno comercial a través de cambios en la legislación que permitan contar con seguridad jurídica sobre a la transmisión de conocimiento. Por último, la entrada de multinacionales, su entrada a la OMC y, en consecuencia, el crecimiento en la de industria, ha polarizado el desarrollo de la innovación en pocas regiones (Chen, 2009; Narula y Dunning, 2010; De la Tour, Glachant y Ménière, 2011; Melaas y Zhang 2016).

\subsection{Aspectos principales de la literatura}

Luego de realizar la revisión literaria de los estudios empíricos de análisis y comparación de SI de China y Alemania, así como de artículos y libros sobre la dinámica de innovación y cambio tecnológico en los países foco, se destacan los siguientes aspectos principales a tomar en consideración tanto respecto a la naturaleza de los SN-R_I como a la metodología que puede ser utilizada para su posterior comparación analítica.

El Estado juega un papel fundamental en la generación de políticas que facilitan la generación y difusión de la innovación mediante la articulación del conocimiento y de las rutinas que se producen a lo largo de un SI(Allen, 2013; Sastre, 2015). De esta forma, la configuración de los actores involucrados en el proceso de innovación -instituciones, empresas, centros de investigación y universidades- se desarrolla como un proceso evolutivo, estructural y enfocado en resultados que requiere tiempo (Fagerberg, Srholec y Verspagen, 2010; Lewis, 2013; Ordóñez, 2017). Esto se evidenció en ambos casos estudiados, particularmente en el caso de Alemania, donde se vinculó a las raíces del actual SI en los procesos de reformas generados en el siglo XIX (Allen, 2015). Similarmente, los estudios del SN-R_I de China destacaron que el cambio del modelo económico nacional y las políticas puestas en marcha dinamizaron la vinculación de sectores responsables del proceso de innovación (Castellani, Jimenez y Zanfei, 2013; Melaas y Zhang, 2016). 
De esta forma, se resalta que la configuración del SI está determinada por el contexto histórico y socio-económico de cada país y región (Liu y White, 2011; Fagerberg, Martin y Andersen, 2013; Sastre, 2015). No obstante, dicho entorno puede direccionarse con políticas que vayan de la mano de las necesidades y capacidades estatales. Así, en el caso alemán, el éxito de sus políticas se debió al énfasis que colocó en sectores históricamente competitivos, como la química y farmacéutica (Hommes, Mattes y Triebe, 2011). Similarmente, las políticas de inversión en I+D se priorizaron para áreas y regiones donde era más probable generar innovación dados los recursos -humanos, materiales y conocimientos previos- manejados durante cada época. En China, igualmente, las políticas se han centrado en la apropiación tecnológica para dinamizar la producción (De la Tour, Glachant y Ménière, 2011; Castellani, Jimenez y Zanfei, 2013; Melaas y Zhang 2016)). De este modo, se busca optimizar el uso de la mano de obra y la adaptación de bienes a la demanda interna y externa dentro del entorno regional inmediato y global.

Además, dado que la competitividad empresarial depende de la capacidad para explotar un invento e innovar, dicha capacidad debe estar en constante estudio y evolución (a partir de la retroalimentación y modificación constante) para permitir cambios en las estrategias empresariales y gubernamentales sobre un bien (Lester, 2004; Kenney y Patton, 2011; Sastre, 2015; Lundvall, 2016). En este caso, los objetivos de innovación de China y Alemania se posicionan acorde con los cambios tecnológicos y espaciales de la globalización. Por un lado, el gobierno chino apunta al desarrollo de una industria manufacturera y de tecnologías de información y comunicación a gran escala. Mientras que, por otro lado, el gobierno alemán vislumbra el desarrollo de tecnologías de última generación que den solución a los desafíos globales -como el cambio climático, la nutrición y la movilidad y la seguridad- en su estrategia tecnológica para 2020 (Hommes, Mattes y Triebe, 2011; Melaas y Zhang, 2016).

Otro aspecto a considerar en el análisis del SN-R_I es la apertura comercial y descentralización del gobierno (Liu y White, 2001; Carlsson, et al. 2002). En el caso de Alemania oriental y China comunista, se vio que la falta de apertura comercial y centralización excesiva del gobierno impedían el desarrollo tecnológico y financiero, debido a que las empresas públicas no contaban con incentivos suficientes para invertir en I+D y poner en marcha innovaciones. Sin embargo, las contrapartes de economía occidental de ambos modelos mostraron la importancia de la inversión privada en el proceso de l+D e innovación. Además, las reformas de descentralización del gobierno alemán en Estados federales facilitaron las tareas de identificar y dirigir los esfuerzos comerciales e innovadores en los sectores económicos de particular capacidad productiva y oportunidad de innovar. Así también, con el caso de China se corrobora la necesidad que tiene la innovación de alimentarse del sector empresarial y que no precise de financiamiento netamente estatal (Shapira y Youtie, 2010).

Las vinculaciones entre actores, de igual manera, son el aspecto primordial que caracteriza a un SI maduro o emergente y su posterior desenvolvimiento en la generación y transmisión de conocimiento (Arocena y Sutz, 2002; Soete, Verspagen y ter Weel, 2010) Este aspecto se reveló como uno de los principales desafíos actuales del SN-R_I chino, el cual cuenta con enlaces emergentes de vinculación débil en comparación con las redes de las instituciones alemanas y estadounidenses, un contexto socioeconómico en reconfiguración con su negociación y entrada al GAAT/OMC; y una dependencia significativa por conocimiento extranjero, propio de su estrategia imitativa (Liang, 2002; Chen, 2009; Hommes, Mattes y Triebe, 2011; Melaas y Zhang, 2016). En ese sentido, aunque el gobierno chino tiene avances significativos en la vinculación entre empresas y centros de investigación nacionales con multinacionales, todavía no cuenta con capacidad de apropiación tecnológica lo suficientemente desarrollada para pasar de una estrategia empresarial 
imitativa a una radical (De la Tour, Glachant y Ménière, 2011; Abdi y Aulakh, 2012). Solo así el SNI chino podría ganar competitividad internacional y beneficiarse de los clústers internacionales conformados por las multinacionales en su territorio (Sastre, 2015; Melaas y Zhang, 2016).

Respecto al análisis comparado de SI, los estudios presentaron una visión neoschumpeteriana y de enfoque en resultados de tres ejes que permiten contrastar políticas de innovación, grados de vinculación y eficiencia de instituciones y actores, y actividades fundamentales de los SN-R_I contrastados (Nelson, 1993; Fagerberg, Martin y Andersen, 2013). Al respecto, el estudio más representativo fue el de Liu y White (2001) en el que propusieron un marco analítico de referencia para evaluar distintos modelos económicos. De dicho trabajo se desprendieron otros dos tipos de análisis que mantenían el enfoque sistémico de tres ejes, aunque no fueron desarrollados a profundidad. Se considera, por tanto, que un análisis comparado de SN-R_I debe tratar dichos ejes para medir factores como la intensidad de la inversión en $\mathrm{I}+\mathrm{D}$, la calidad de la inversión, los inputs y outputs, así como el entorno regional, nacional y global en el que dichas políticas toman lugar.

De esta forma, dentro de las comparaciones de los SN-R_I de Alemania y China se destacaron las políticas gubernamentales que promueven el desarrollo de sistemas educativos especializados y su vinculación con la actividad comercial. Asimismo, se enfatizó en los programas de patrocinio de I+D en sectores especializados y el desempeño de la inversión en patentes y crecimiento económico (Hommes, Mattes y Triebe, 2011). Es importante destacar que estas comparaciones permiten a los tomadores de decisión, tanto públicos como privados, identificar estrategias ventajosas que resulten en procesos de cooperación con otros actores, la correcta importación y adaptación de políticas exitosas en otros países, e incluso tácticas de defensa de activos críticos -como el conocimiento tácito, las patentes de innovaciones radicales, y los procesos de producción, modificación y comercialización- frente a otros actores. Esto, dado que el resultado de la inversión en I+D también debe ser considerado a partir del grado de competitividad de un estado y de sus empresas en mercados nacionales e internacionales (Shapira y Youtie, 2010).

Finalmente, con relación a la identificación de actores y fuentes de información para la comparación de SN-R_I, los estudios enfatizaron el uso de datos provenientes de fuentes gubernamentales y privadas. Así, carteras de Estado a cargo de la tecnología e innovación, cámaras de comercio, centros de investigación y encuestas nacionales de innovación constituyen fuentes primaras clave en el proceso de recopilación de información nacional. Igualmente, fuentes como la OECD, los rankings pertenecientes a la Fundación de Tecnologías de Información e Innovación (ITIF) y el Índice Global de Innovación (GIL) contribuyen con indicadores mundiales clave para dirigir o verificar los datos nacionales. Por último, dentro de los desafíos restantes se destaca la dificultad para conseguir información actualizada China en medios oficiales, puesto que la página del Ministerio de ciencia y tecnología maneja datos hasta 2006 y el resto se los debe identificar en otros sitos gubernamentales de manera esparcida y similarmente al caso alemán, en la lengua nativa del país.

\section{Conclusiones}

El estudio de la innovación desde una visión nacional-regional sistémica presenta una oportunidad para mejorar la comprensión de los tomadores de decisión, tanto públicos como privados, sobre las dinámicas socio-económicas y tecnológicas a considerar al momento de establecer políticas de desarrollo y proyección financiera. Dentro el presente trabajo se ha 
buscado evidenciar las aproximaciones teóricas y metodológicas usadas en estudios empíricos que comparan el SN-R_I en Alemania y China a modo de contribuir en futuras investigaciones.

La revisión de la literatura en torno a seis ensayos principales sobre SI evidenció en primer lugar que existen avances en el estudio del SN-R_I bajo la perspectiva evolutiva neoschumpeteriana y el enfoque a resultados de los modelos de partida. Sin embargo, se destacó, también, la complejidad del estudio sistémico de la innovación. Este análisis requiere de la consideración del contexto socio-económico, histórico y geográfico a nivel nacional y regional, de una estructuración de las vinculaciones entre organizaciones del SN-R_I y de una revisión de las políticas públicas de innovación y sus resultados. Asimismo, la investigación de los SN-R_I de distintos países puede ayudar a procesos de integración económica y cooperación interestatal, así como a la adaptación e incorporación de prácticas exitosas de otros SN-R_I para continuar desarrollando la capacidad competitiva nacional y regional.

Dentro del análisis se estableció que China y Alemania cuentan con un SN-R_I en diferentes etapas de desarrollo. Dicha peculiaridad complejiza su comparación sistemática bajo perspectivas clásicas. No obstante, con la ayuda de nuevos marcos de comparación sobre las visiones de políticas y vinculaciones entre actividades y actores del SN-R_I es mucho más factible realizar un estudio comparado. Al respecto, el modelo de análisis central que ha perfilado los nuevos enfoques de estudios comparados de SN-R_I es el presentado por de Liu y White en 2001, dentro de un estudio que distingue las vinculaciones económicas de China antes y después de las reformas económicas. Dicho modelo de tres ejes sistémicos -estructura, dinámicas y desempeño- es tomado como base para análisis más contemporáneos de SI estatales y regionales.

Dentro de los casos estudiados, se destaca el éxito del sistema alemán. En este, las reformas históricas en las políticas educativas e industriales repercutieron en avances dentro de los procesos de desarrollo tecnológico e investigación especializada. Además, esta arquitectura de innovación se ha desarrollado hasta contar con complejas redes de interconexión entre agentes innovadores y centros de investigación en sectores y regiones, lo que facilita el proceso de aprendizaje para la generación, difusión y retroalimentación del conocimiento. Asimismo, en los estudios sobre Alemania, se revela un enfoque de investigación altamente especializado en sectores tradicionalmente fuertes, como la industria automotriz, la química, los materiales de construcción y farmacéutica, lo que muestra una lógica de generación de innovación de manera incremental. Dichos sectores innovan, en esencia, a partir de medianas y pequeñas empresas cuyos fondos para el proceso de I+D provienen del sector privado, pero sus investigadores provienen de centros de investigación y universidades públicas de alto nivel educativo. Sobre los desafíos del SN-R_I alemán, los estudios sugieren mejorar el nivel de especialización tecnológica para ubicarlo al nivel de Estados Unidos y facilitar un ambiente de emprendimiento con el fin de lograr más innovaciones de carácter radical a nivel regional, nacional y global.

Respecto al caso del SN-R_I de China, los estudios resaltan el papel del Estado en cuanto a políticas de apertura comercial privada, financiación de centros de investigación y universidades y a la adquisición tecnológica para dinamizar la producción y comercialización de su industria. No obstante, se insiste en la fase emergente del SN-R_I chino, en cuanto a las configuraciones de sus vínculos entre agentes y regiones, lo cual envuelve vinculaciones todavía débiles entre diversos sectores, como universidades, empresas privadas e instituciones reguladoras. Además, se hace hincapié en el rol de las multinacionales en la economía china y su modelo de innovación. Dichas firmas generan alianzas estratégicas locales, regionales y globales que permiten la transmisión de conocimiento básico en el proceso industrial, pero que a su vez dependen del desarrollo empresarial por medio de estrategias de imitación y complementación entre dichas empresas. Finalmente, los dos desafíos más importantes para el SN-R_I de China son generar un ambiente empresarial estable mediante normas jurídicas, reducción de la desigualdad social, así como ajustar los incentivos de I+D que relacionan a los actores del sistema, tanto a nivel nacional, 
regional y global.

Finalmente, el presente trabajo dio a conocer diversas fuentes de datos e información para analizar los SN-R_I de Alemania y China. Estas incluyen los ministerios a cargo de las políticas de ciencia, tecnología e innovación, las cámaras de comercio, los centros de investigación y encuestas nacionales de innovación como fuentes primarias. Al mismo tiempo, estudios como los de la OECD, los rankings de la Fundación de Tecnologías de Información e Innovación (ITIF) y el Índice Global de Innovación (GIL) representan fuentes secundarias que favorecen a la comparación estatal de SN-R_I.

\section{Referencias bibliográficas}

- Fagerberg, J., Martin, B. y Andersen, E. (2013). Innovation Studies: Towards a New Agenda. En J. Fagerberg, B. Martin y E. Andersen. (Ed.), Innovation Studies: Evolution and Future challenges (pp. 1-17). Nueva York, Estados Unidos: Oxford University Press.

- Soete, L. Verspagen, B. y ter Weel, Bas. (2010). Systems of Innovation. En B. Hall, N. Rosenberg. (Eds.), The Handbook of the Economics of Innovation (pp. 11591180). Oxford: Elsevier B. V.

- Edquist, C. (2005). Systems of innovation: Perspectives and Challenges. En J. Fagerberg, D. Mowery y R. Nelson. (Ed.), The Oxford Handbook of Innovation (pp. 181-208). Nueva York, Estados Unidos: Oxford University Press.

- Fagerberg, J. (2005). Innovation: A Guide to the Literature. En J. Fagerberg, D. Mowery y R. Nelson, (Eds.), The Oxford Handbook of Innovation (pp. 1-26). Nueva York, Estados Unidos: Oxford University Press.

- Mokyr, J. (2010). The Contribution of Economic History to the Study of Innovation and Technical Change: 1750-1914. En B. Hall, N. Rosenberg. (Eds.), The Handbook of the Economics of Innovation (pp. 11-50). Oxford: Elsevier B. V.

- Freeman, C. (1987). Technology Policy and Economic Performance: Lessons from Japan. Londres: Pinter.

- Sastre, J. F. (2015). Economía neo-schumpeteriana, innovación y política tecnológica. Cuadernos de Economía 38, pp. 79-89.

- Lundvall, B. (1992). National Systems of Innovation: Towards a Theory of Innovation and Interactive Learning. Londres: Pinter.

- Nelson, R. y Rosenberg, N. (1993). Technical Innovation and National Systems. En Nelson, R. (Ed.), National Systems of Innovation: a Comparative Study (pp. 3-21). Oxford: Oxford University Press.

- Verspagen, B. (2005). Innovation and Economic Growth. En J. Fagerberg, D. Mowery y R. Nelson, (Eds.), The Oxford Handbook of Innovation (pp. 487-513). Nueva York, Estados Unidos: Oxford University Press.

- Arocena, R. y Sutz, J. (2002). Innovation systems and developing countries. DRUID 
(Danish Research Unit for Industrial Dynamics) Working Paper 2 (05).

- Allen, G. (2013). What is International Political Economy. En D. Balaam y B. Dillman (Eds.), Introduction to International Political Economy. New York: Routledge.

- Liu, X. y White, S. (2001). Comparing innovation systems: a framework and application to China's transitional context. Research Policy 30, pp. 1091-1114. d

- Chaminade, C., Lundvall, B., Vang, J. y Joseph, K.J. (2010). Innovation policies for development: towards a systemic experimentation based approach. Papers in Innovation Studies 2010/1, Lund University, CIRCLE.

- Melaas, A., y Zhang, F. (2016). National Innovation Systems in the United States and China: A Brief Review of the Literature. Medford: Tufts University.

- Keck, O. (1993). The National System for Technical Innovation in Germany. En Nelson, R. (Ed.), National Innovation Systems: a comparative analysis (pp. 115-157). Nueva York: Oxford University Press.

- Allen, M. (2015). The National Innovation System in Germany. En Narayanan V. y O'Connor G. (Ed.), Wiley Encyclopedia of Management: Technology and Innovation Management (pp. 375-389). Wiley.

- Thelen, K. (2004). How Institutions Evolve: The Political Economy of Skills in Germany, Britain, the United States and Japan. Cambridge, Reino Unido: Cambridge University Press.

- Culpepper, P. y Finegold, D. (1999). The German Skills Machine: Sustaining Comparative Advantage in Global Economy. Oxford, Reino Unido: Berghahn Books.

- Whitley, R. (2007). Business Systems and Organizational Capabilities: The Institutional Structuring of Competitive Competences. Oxford, Reino Unido: Oxford University Press.

- Hommes, C., Mattes, A. y Triebe, D. (2011). Research and Innovation Policy in the U.S. and Germany: A Comparison. Belin: DIW Berlin.

- Kroll, H y Frietsch, R. (2010): Recent Trends in innovation policy in Germany. En Frietsch, R. y Schüller, M. (Eds.), Competing for global innovation leadership: Innovation systems and policies in the USA, Europe and Asia. (pp. 73-92). Stuttgart: Fraunhofer Verlag.

- American Institute for Contemporary German Studies (AICGS). (2006). AICGS Policy Report 26. Part one: Innovation in the United States and Germany. Estados Unidos: The John Hopkins University.

- Wu, X., Revi, A. y Doshi, H. (2009). The Comparison of China and India: National Innovation System. Communications, pp. 7-12. India China Institute.

- Patel, P. y Pavitt, K., (1998). National System of Innovation Under Strain: The Internationalization of Corporate R\&D. Electronic working Paper series, Paper No. 22, http://www.sussex.ac.uk/spru/ 
- Anderson, E. y Lundvall, E. (1997). National innovation systems and the dynamics of the division of labor. En Edquist, C. (Ed.), Systems of Innovation: Technologies, Institutions and Organizations. (pp. 242-265). Londres: Pinter.

- Castellani, D., Jimenez, A. y Zanfei, A. (2013). How remote are R\&D labs? Distance factors and international innovative activities. Journal of International Business Studies (2013), pp. 1-27.

- Fagerberg, J., Srholec, M. y Verspagen, B. (2010). Innovation and economic Development. En B. Hall, N. Rosenberg. (Eds.), The Handbook of the Economics of Innovation (pp. 833-872). Oxford: Elsevier B. V.

- Abdi, M., y Aulakh, P. (2012). Do country-level institutional frameworks and interfirm governance arrangements substitute or complement in international business relationships? Journal of International Business Studies, 43(5), pp. 477-497.

- Carlsson, B., Jacobsson, S, Holménb, M. y Rickne, A. (2002). Innovation systems: analytical and methodological issues. Research Policy 31 (2), pp. 233-245.

- Guo B. y Guo J.J. (2011). Patterns of technological learning within the knowledge systems of industrial clusters in emerging economies: Evidence from China. Technovation 31 (2-3), pp. 87-104.

- Cai, Y., Cui, L. y Pugh, R. (2015). Enabling Conditions for Regional Triple Helix Systems. Triple Helix 4 (4), pp. 19-23.

- De la Tour, A., Glachant, M. y Ménière, Y. (2011). Innovation and international technology transfer: The case of the Chinese photovoltaic industry. Energy Policy 39 (2), pp. 761-770.

- Gallagher, K. y Zhang F. (2016). Innovation and Technology Transfer Through Global Value Chains: Evidence from China's PV Industry. Energy policy 94, pp. 191203.

- Todo, Y., Zhang, W. y Zhou, L. (2006). Intra-Industry Knowledge Spillovers from Foreign Direct Investment in R\&D: Evidence from a Chinese Science Park. SSRN Electronic Journal, pp. 1-34.

- Narula, R. y Dunning, J.H. (2010). Multinational enterprises, development and globalization: Some clarifications and a research agenda. Oxford Development Studies. 38, pp. 263-287.

- Carey, D., Hill, C. y Kahin, B. (2012). Strengthening Innovation in the United States. OECD Economics Department Working Papers No. 1001, OECD Publishing.

- Lewis, J. (2013). Green Innovation in China: China's wind power industry and the global transition to a low-carbon economy. Nueva York: Columbia University Press.

- Lester, R. y Piore, M. (2004). Innovation: The Missing Dimension. Cambridge: Harvard University Press.

- Kenney, M., y Patton, D. (2011). Does inventor ownership encourage university re- 
search-derived entrepreneurship? A six university comparison. Research Policy 40 (8), pp. 1100-1112.

- Shapira, P., y Youtie, J. (2010). The Innovation System and Innovation Policy in the United States. En R. Frietsch, y M. Schüller, (Eds.), Competing for Global Innovation Leadership: Innovation Systems and Policies in the USA, EU and Asia, pp.529. Stuttgart: Fraunhofer IRB Verlag.

- Ordóñez, S. (2017). Sistemas de Innovación y Conocimiento: El Caso de Jalisco, México. Revista Problemas del Desarrollo 191 (48), pp. 161-184.

- Vivares, E., Torres P., y Cvetich K. (2014). Enfoques y cárceles conceptuales en el entendimiento de los Nuevos Regionalismos Latinoamericanos. En A. Bonilla e I. Álvarez (Eds.), Desafíos estratégicos del regionalismo contemporáneo: CELAC e Iberoamérica, pp. 21-46. San José, Costa Rica: FLACSO.

- Quintero-Campos, L. (2010). Aportes teóricos para el estudio de un Sistema de innovación. Innovar 20(38), pp. 57-76.

- Liu X., Han S., y Chen A. (2014). Innovation System and Inequality Reduction in China. En M. Couto, M. Scerri y R. Maharaih (Eds.), BRICS National Systems of Innovation: Inequality and Development Challenges, pp. 190-237. Reino Unido: Routledge.

- Lundvall B. (2016). National Systems of Innovation: Towards a Theory of Innovation and Interactive Learning. En B. Lundvall (Ed.), The Learning Economy and the Economics of Hope, pp. 85-106. Nueva York: Anthem Press.

- Chen, C. (2009). China's economy after WTO accession: an overview. En C. Chen (Ed.), China's Integration with the Global Economy: WTO Accesion, Foreign Direct Investment and International Trade, pp. 1-18. Reino Unido: Edward Elgar Publishing Limited.

- Liang, W. (2002). China's WTO Negotiation Process and its Implications. Journal of Contemporary China 11 (33), pp. 683-719.

- Kirshner, J. (2009). Realist political economy: traditional themes and contemporary challenges. En M. Blyth (Ed.), Routledge Handbook of International Political Economy (IPE), pp. 36-47. Nueva York: Routledge.

- Keohane, R., y Nye J. (2012). Power and Interdependence. Cuarta edición, Nueva York: Longman.

- Miguelez, D. (2017). La industria 4.0 en Alemania. Oficina Económica y Comercial de la Embajada de España en Berlín. Berlín: ICEX España Exportación e Inversiones.

- Wübbeke, J., Meissner M., Zenglein M., Ives J., y Conrad B. (2016). Made in China 2015: The making of a high-tech superpower and consequences for industrial countries. MERICS Papers on China n2, Berlin: Mercator Institute for China Studies (MERICS). 
